\title{
STRONG-FIELD PHENOMENA IN ATOMS - QUASICLASSICAL APPROACH
}

\author{
M.V. Fedorov \\ General Physics Iristitute, Russian Academy of Sciences, Moscow, Russia
}

A quasiclassical (WKB) approach is used to construct a theory of atomic transitions induced by a strong light field. This approach is used to find the "Coulomb-Volkov" solutions of the Schrödinger equation in which both the Coulomb and light fields are taken into account. These solutions are shown to be applicable in a region of low light frequencies, low electron energies and angular momenta. The found solutions are used to describe two kinds of processes: strong-field photoionization from highly excited (Rydberg) atomic levels, and field-assisted electron-ion scattering. In the photoionization problem the strong-field complex quasi-energies of an atom are found. A problem of the strong-field stabilization of an atom, as well as the expected behavior of the ionization time in its dependence on the light field strength are discussed.

PACS numbers: $32.80 . \mathrm{Fb}, 32.80 . \mathrm{Rm}$

\section{Introduction}

Already for many years a problem of an atomic electron in a strong light field attracts a significant attention of scientists, both theorists and experimentalists, working in the domain of the physics of light-atom interactions. From the theoretical point of view this problem is rather interesting and rather difficult. Even in the simplest case of the hydrogen atom a problem of an electron motion in a pure Coulomb potential in the presence of a strong light field has no exact analytical solution and can be solved only approximately. During several last years such approximate theories were extended to predict many interesting and unusual strong-field phenomena. One of them is the field-induced stabilization of atoms, or suppression of photoionization or multiphoton ionization in a strong-field limit. There are several models predicting such an effect [1]. In this talk the main attention will be devoted to the so-called interference stabilization of Rydberg atoms [2] (see also the list of references in this review paper). In accordance with the existing ("old") theory the effect is explained by $A$-type Rydberg-Rydberg transitions via the continuum resulting in the secondary coherent repopulation of the Rydberg levels. Subsequent transitions from these levels to the continuum interfere 
with each other causing a suppression of photoionization or stabilization of an atomic population on the Rydberg levels. Not dwelling here upon any details of this "old" theory, let us mention only that the expected critical field $\varepsilon_{\mathrm{c}}$ beginning from which the interference stabilization effect is expected to occur is estimated as $\varepsilon_{\mathrm{c}} \sim \omega^{5 / 3}$ where $\omega$ is the light frequency, $\omega \ll 1$ (both $\varepsilon_{0}$ and $\omega$ in atomic units). IIere and below we assume that $\omega$ is larger than the Rydberg electron binding energy, $\omega>\left|E_{n}\right| \ll 1$ where $E_{n}=-1 / 2 n^{2}$, and $n$ is the atomic electron principal quantum number, $n \gg 1$. This estimate follows from the so-called quasiclassical (or WKB) approach widely used and discussed below. On the other hand, the "old" theory of the strong-field photoionization from the Rydberg levels exploits also several other approximations applicability of which hardly can be proved rigorously. Some of these approximations are the well-known rotating wave approximation (RWA), the so-called "pole" approximation, the flat continuum approximation, adiabatic elimination of the continuum, the model of essential states, and so on. The main goal of this talk is to present a theory free from most of these approximations, and based mostly on the quasiclassical approach.

To complete the introduction to this "new" strong-field theory, let us remind briefly how the quasiclassical approach works in the weak-field limit $[3,4]$. This approximation is known to be valid in a region of positive or negative electron energies close to the ionization threshold: $\left|E_{n}\right| \ll 1$ and $E \ll 1$ where $E>0$ is the atomic electron energy in the continuum. In this case the bound and free radial atomic electron wave functions are given by [5]:

$$
\begin{aligned}
& \chi_{n}(r)=C_{n}\left[C_{n}\right]^{-1 / 2} \sin \left[\int_{0}^{r} \mathrm{~d} r^{\prime} p_{n}\left(r^{\prime}\right)+\pi / 4\right], \\
& \chi_{E}(r)=C_{E}\left[P_{E}(r)\right]^{-1 / 2} \exp \left[\mathrm{i} \int_{0}^{r} \mathrm{~d} r^{\prime} p_{E}\left(r^{\prime}\right)\right],
\end{aligned}
$$

where $C_{n}$ and $C_{E}$ are the normalization constants, and $p_{n}(r)$ and $p_{E}(r)$ are the quasiclassical momenta.

$$
p_{n}(r)=\left[2\left(E_{n}+1 / r\right)\right]^{1 / 2}, \quad p_{E}(r)=[2(E+1 / r)]^{1 / 2} .
$$

The rate of bound-free transitions in the weak-field theory is determined by the bound-free matrix elements $V_{n E}$ (via the Fermi golden rule) where $V_{n E}=$ $\left\langle\chi_{n}\left|(1 / 2) d \varepsilon_{0}\right| \chi_{E}\right\rangle$, and $d$ and $\varepsilon_{0}$ are the atomic dipole moment and the light field strength amplitude. In the quasiclassical region $\left(\left|E_{n}\right| \ll 1, E \ll 1\right)$ the quasiclassical momenta $p_{n}(r)$ and $p_{E}(r)$ of Eq. (2) can be expanded in powers of $r\left|E_{n}\right| \ll 1$ and $r E \ll 1$ to give

$$
V_{n E}=2^{-3 / 2} C_{n} C_{E} \varepsilon_{0} \int_{0}^{\infty} \mathrm{d} r r^{3 / 2} \exp \left(-\mathrm{i} \omega r^{3 / 2}\right),
$$

where the energy conservation rule $E=E_{n}+\omega$ is taken into account. Equation (3) yields immediately the main quasiclassical estimate of the bound-free matrix element $V_{n E} \sim \varepsilon_{0} / \omega^{5 / 3} n^{3 / 2}$. This equation shows also that the main contribution to the quasiclassical bound-free transitions is given by a vicinity of the quasiclassical electron-nucleus distance $r \sim r_{\mathrm{q}}$ where

$$
r_{\mathrm{q}}=\omega^{-2 / 3} \text {. }
$$


By an assumption [4] this quasiclassical length has to be much shorter than the Rydberg orbit size $r_{\mathrm{m}}=2 n^{2} \sim 1 / \omega$ (to justify the used expansion in powers of $r\left|E_{n}\right| \ll 1$ and $\left.r E \ll 1\right)$. The condition $r_{\mathrm{q}} \ll r_{\mathrm{m}}$ detcrmines the main small parameter of the quasiclassical theory $\omega^{1 / 3} \ll 1$. In other words, the light frequency $\omega$ has to be small enough for the quasiclassical approximation to be valid. In reality inequality $\omega^{1 / 3} \ll 1$ is satisfied in the IR, or longer wavelength regions. One can check easily that under the condition $\omega^{1 / 3} \ll 1$ the centrifugal term $l(l+1) / r^{2}$ in the Schrödinger equation estimated at $r \sim r_{\mathrm{q}}$ is much smaller than the Coulomb energy $1 / r$ if only the angular momentum quantum number $l$ is not too large, $l \ll \omega^{-1 / 3}$. For such states the centrifugal term can be dropped and the matrix element $V_{n E}$ appears to be independent of the electron orbital momentum [4].

\section{Strong-field quasiclassical quasi-energy solutions of the Schrödinger equation}

Let us consider now the strong-field case by assuming that $\varepsilon_{0}$ can be either large, or small as compared to, or of the order of $\varepsilon_{\mathrm{c}}=\omega^{5 / 3}$. Let us assume that all the main features of the quasiclassical approximation described above still remain valid in the strong-field theory. We will assume in particular that in the strong-field theory for low-angular momentum states the centrifugal term can be dropped from the time-dependent Schrödinger equation. After such a simplification an equation for the radial part of the electron wave function $\chi(r)=r \Psi(r)$ takes a form

$$
\mathrm{i} \frac{\partial \chi}{\partial t}=\left[-\frac{1}{2} \frac{\partial^{2}}{\partial r^{2}}+\frac{1}{r}+\varepsilon_{0} r \sin (\omega t)\right] \chi
$$

where in principle both $\chi$ and $\Psi$ depend parametrically on the angle $\theta$ between the vectors $\varepsilon_{0}$ and $r$ (rigorously $\varepsilon_{0}$ in Eq. (5) has to be understood as $\varepsilon_{0} \cos \theta$ ). Let us present the quasi-energy solutions of Eq. (5) in the form

$$
\begin{aligned}
& \chi_{\gamma \pm}=[2(\gamma+1 / r)]^{-1 / 4} \exp \left\{-\mathrm{i} \gamma t \pm \mathrm{i} \int_{0}^{r} \mathrm{~d} r^{\prime}\left[2\left(\gamma+1 / r^{\prime}\right)\right]^{1 / 2}\right\} \\
& \times \exp \left\{-\mathrm{i}\left[\left(\varepsilon_{0} / \omega\right) r \cos (\omega t)+\left(\varepsilon_{0} / 2 \omega\right)^{2} t+\left(\varepsilon_{0}^{2} / 8 \omega^{3}\right) \sin (2 \omega t)-f_{\gamma \pm}(r, t)\right]\right\},
\end{aligned}
$$

where $\gamma$ is the quasi-energy and the signs "+" and "-" correspond to the rightand left-hand direction propagating traveling quasiclassical waves. Each of the functions $\chi_{\gamma \pm}$ can be considered as a product of two factors. The first of them includes the pre-exponential factor and the first exponent on the right-hand side of Eq. (6). This part reminds closely the field-free quasiclassical wave functions [1]. On the other hand, the second exponent on the right hand side of Eq. (6) reminds some components of the nonrelativistic Volkov function. In principle Eq. (6) can be considered as a definition of a new unknown function $f_{\gamma \pm}(r, t)$ introduced instead of $\chi_{\gamma \pm}(r, t)$. By using this definition and substituting $\chi_{\gamma \pm}$ of Eq. (6) into the Sclirödinger equation (5) we can derive an exact equation for $f_{\gamma \pm}(r, t)$ which is too complicated to be written explicitly here. But this equation can be significantly simplified under the assumption that the most important region of $r$ is $r \sim r_{\mathbf{q}}$ 
(Eq. (4)). Then many terms in the exact equation for $f_{\gamma \pm}(r, t)$ can be dropped as having a higher order in a small parameter $\omega^{1 / 3}$ to give

$$
\frac{\partial f}{\partial t}= \pm[2(\gamma+1 / r)]^{1 / 2}\left[\frac{\partial f}{\partial r}-\left(\varepsilon_{0} / \omega\right) \cos (\omega t)\right]
$$

where $f=f_{\gamma \pm}(r, l)$. Equation (7) is already simple enough to be solved and to yield

$$
f(r, t)= \pm\left(\varepsilon_{0} / \omega\right) \int_{0}^{t} \mathrm{~d} t^{\prime} \cos \left(\omega t^{\prime}\right) v_{\mathrm{cl}}\left( \pm \tau(r)+t-t^{\prime}\right)
$$

where $\tau(r)$ is the time it takes for a classical particle with an energy $\gamma$ to move in the Coulomb field from the point $r=0$ to $r$ :

$$
\tau(r)=\int_{0}^{r} \mathrm{~d} r^{\prime}\left[2\left(\gamma+1 / r^{\prime}\right)\right]^{-1 / 2}
$$

$v_{\mathrm{cl}}(t)=\dot{r}_{\mathrm{cl}}(t)$ and $r_{\mathrm{cl}}(t)$ are the velocity and time-dependent radial coordinate of a classical electron on its field-free Coulomb trajectory

$$
v_{\mathrm{cl}}(t)=\left\{\left[2\left[\gamma+1 / r_{\mathrm{cl}}(t)\right]\right\}^{1 / 2} .\right.
$$

Now the strong-field quasiclassical quasi-energy solutions (6) $\chi_{\gamma \pm}=r \Psi_{\gamma \pm}$ of the Schrödinger equation (5) take a form

$$
\begin{aligned}
\chi_{\gamma \pm}(r, t) & =[2(\gamma+1 / r)]^{-1 / 4} \exp \left\{-\mathrm{i} \gamma t \pm \mathrm{i} \int_{0}^{r} \mathrm{~d} r^{\prime}\left[2\left(\gamma+1 / r^{\prime}\right)\right]^{1 / 2}\right\} \\
& \times \exp \left\{-\mathrm{i}\left[\left(\varepsilon_{0} / \omega\right) r \cos (\omega t)+\left(\varepsilon_{0} / 2 \omega\right)^{2} t+\left(\varepsilon_{0}^{2} / 8 \omega^{3}\right) \sin (2 \omega t)\right.\right. \\
& \left.\left. \pm\left(\varepsilon_{0} / \omega\right) \int_{0}^{t} \mathrm{~d} t^{\prime} \cos \left(\omega t^{\prime}\right) v_{\mathrm{cl}}\left( \pm \tau(r)+t-t^{\prime}\right)\right]\right\}
\end{aligned}
$$

These functions can be considered as a quasiclassical analog of the Volkov functions. They are valid in the low-energy low-frequency region $|\gamma| \ll 1, \omega \ll 1$. IIowever, if in the Volkov functions an atomic potential is ignored completely, in the functions $\chi_{\gamma \pm}(r, t)$ of Eq. (11) both the electron-light and the electron-Coulomb interactions are taken into account (in the quasiclassical approximation).

It should be noted that an approach similar to that described above, though in a different and more complicated form, was used earlier by Bersons [6].

\section{Strong-field photoionization from Rydberg levels}

Let us apply now the derived expressions for the quasiclassical quasi-energy wave functions $\chi_{\gamma \pm}(r, t)$ (Eq. (11)) to a problem of a strong-field photoionization from the Rydberg levels. In this work the formulated problem will be considered as an eigenvalue problem, and the corresponding eigenvalues (i.e., complex quasi-energies) will be found from a generalized Bohr-Sommerfeld quantization rule derived below. As usual, this quantization rule follows from the reasonably formulated boundary conditions. Not dwelling here upon any details let us mention only that the most important difference with the field-free case arises from the region of small $r\left(r \leq r_{\mathrm{q}}\right)$. In this region Eqs. (8) and (11) can be significantly simplified. In particular, $\tau(r)$ in the argument of $v_{\mathrm{cl}}\left( \pm \tau(r)+t-t^{\prime}\right)$ can be put 
equal to zero. The classical velocity itself can be replaced by its small-t singular expression $v_{\mathrm{cl}}(t) \approx(4 / 3 t)^{1 / 3}$, and the upper limit of the integral over $t^{\prime}$ in $\mathrm{Eq}$. (11) can be replaced by $\infty$ to give

$$
f(0, t) \approx \pm \zeta \sin (\omega t+\pi / 6) .
$$

In the same region $(r \rightarrow 0)$ the quasi-energy wave function $\chi_{\gamma}(r, t)$ obeying the corresponding large- $r$ boundary conditions takes a form

$$
\begin{aligned}
& \chi_{\gamma}(r, t)=(r / 2)^{1 / 4} \exp \left\{-\mathrm{i}\left[\left(\gamma+\left(\varepsilon_{0} / 2 \omega\right)^{2}\right) t+\left(\varepsilon_{0}^{2} / 8 \omega^{3}\right) \sin (2 \omega t)\right]\right\} \\
& \quad \times \sum_{k} J_{k}(\zeta) \sin \left\{-\mu+\pi / 4+\int_{0}^{r} \mathrm{~d} r^{\prime}\left[2\left(\gamma+1 / r^{\prime}\right)\right]^{1 / 2}-k(\omega t+\pi / 6)\right\},
\end{aligned}
$$

where $\zeta=(4 / 3)^{1 / 3} \Gamma(2 / 3) \varepsilon_{0} / \omega^{5 / 3}, \Gamma$ and $J_{k}$ are the gamma- and Bessel-functions [7], and $\mu=\pi / \sqrt{-2 \gamma}$. The most important feature of the result derived (Eq. (13)) consists in the appearance of all the Fourier (or Floquet) structure in a single quasi-energy wave function. Such a function cannot obey the zero boundary at $r=0$ at any $t$. This condition can be fulfilled only if the total wave function has a form of a superposition of $\chi_{\gamma}(r, t)$ of Eq. (13) and of an infinite set of outgoing (reflected) waves with quasi-energies differing from $\gamma$ by $n \omega$, where $n=1,2, \ldots$

$$
\chi_{\text {tot }}=\chi_{\gamma}+\sum_{n} C_{n} \chi_{\gamma+n \omega ;+}
$$

where $C_{n}$ are some unknown constants. It should be noted that Eq. (14) is derived under the assumption that there is no interaction between the quasi-energy level $\gamma$ and any lower lying levels with quasi-energies $\gamma-n \omega$. This simplifying assumption can be valid if $\omega$ is not too large (though is much smaller than one), so that for any $n$ the difference $\gamma-n \omega$ does not approach any real atomic levels. However, if $\omega$ is small enough for the quasiclassical approximation to be well applicable, the levels of energies $\sim \gamma-n \omega$ can belong to the same set of Rydberg levels as $\gamma$. In this case the sum over $n$ in Eq. (14) has to be extended to include negative $n$. Such an extension makes a problem under consideration much more complicated. For this reason in the present analysis we consider a simplified case when all the interactions between the quasi-energy levels $\gamma$ and $\gamma-n \omega$ can be ignored.

Now with the help of the mentioned above small-r boundary condition one can find both the expansion coeflicients $C_{n}$ in Eq. (14) and the dispersion equation for $\mu$ or quasi-energy $\gamma$ (the generalized Bolir-Sommerfeld quantization rule). The latter can be shown to have a form

$$
\exp (-2 \mathrm{i} \mu)=J_{0}(2 \zeta) \text {. }
$$

From this equation one can find easily both the real and imaginary parts of $\mu$ $\left(\mu=\mu^{\prime}+\mathrm{i} \mu^{\prime \prime}\right)$ and of the complex quasi-energy $\gamma=\gamma^{\prime}+\mathrm{i} \gamma^{\prime \prime}$ :

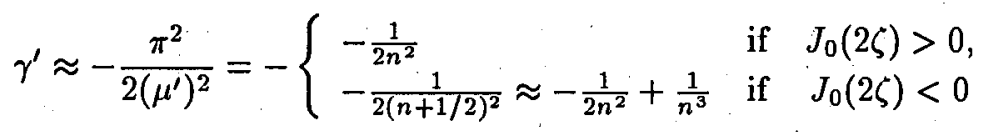

and

$$
\gamma^{\prime \prime} \approx \frac{\pi^{2} \mu^{\prime \prime}}{\left(\mu^{\prime}\right)^{3}} \approx \frac{\ln \left|J_{0}(2 \zeta)\right|}{2 \pi n^{3}}
$$




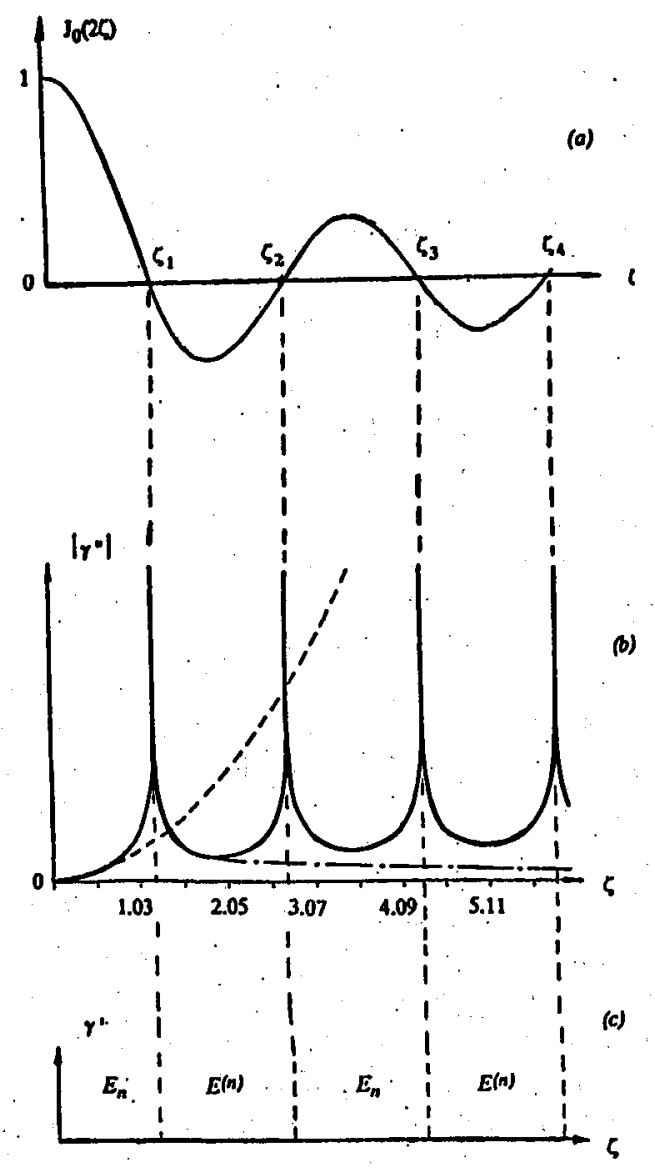

Fig. 1. The zero-order Bessel function (a), and imaginary (b) and real (c) parts of the strong-field quasi-energies vs. the field parameter $\zeta \propto \varepsilon_{0} / \omega^{5 / 3}$.

The results derived are shown in Fig. 1. The dashed and dash-dotted lines correspond to the results of the perturbation theory and of the "old" strong-field theory, respectively. Probably the most interesting prediction of the new strong-field theory concerns a quasiperiodicity of the stabilization-destabilization regions which follow after each other and in which $\left|\gamma^{\prime \prime}\left(\varepsilon_{0}\right)\right|$ falls and grows again (Fig. 1b). The regions of the fastest decay are close to the zero-points $\zeta_{i}(i=1,2, \ldots)$ of the zero-order Bessel function $J_{0}(2 \zeta)$. The minimal values of $\left|\gamma^{\prime \prime}\right|$ are of the order of the inverse classical Kepler period $t_{\mathrm{K}}=2 \pi n^{3},\left|\gamma^{\prime \prime}\right|_{\min } \sim 1 / t_{\mathrm{K}}$. However, it should be noted that a direct connection between $\left|\gamma^{\prime \prime}\right|$ and the ionization time $T_{\mathrm{i}}$ $\left(T_{\mathrm{i}} \approx 1 /\left|\gamma^{\prime \prime}\right|\right)$ occurs only in the weak-field limit when $\left|\gamma^{\prime \prime}\right| \ll 1 / t_{\mathrm{K}}$. In the case $\left|\gamma^{\prime \prime}\right| \geq 1 / t_{\mathrm{K}}$ the "old" theory predicts [8] $T_{\mathrm{i}} \sim t_{\mathrm{K}}$. A similar prediction of the new theory concerning the ionization time is the following: the ionization time is expected to be a falling function of the field strength until $\varepsilon_{0}$ is weaker than $\omega^{5 / 3}$. And then, in the region $\varepsilon_{0}>\omega^{5 / 3}, T_{i}\left(\varepsilon_{0}\right)$ is expected to be stabilized on the level 
$\sim t_{\mathrm{K}}$, maybe with oscillations around $t_{\mathrm{K}}$ with a relative amplitude of the order of one. To confirm these expectations rigorously one has to solve the initial value problem under the same approximations and assumptions as used above, and such a problem is not yet solved.

As for the real parts of the quasi-energies $\gamma^{\prime}$ (Fig. 1c), they can be equal either to the field-free Rydberg energies $E_{n}$ (as long as $J_{0}(2 \zeta)>0$ ) or to the strong-field quasi-energies of the "old" theory [2] $E^{(n)}=\frac{1}{2}\left(E_{n}+E_{n+1}\right)$ if $J_{0}(2 \zeta)<0$.

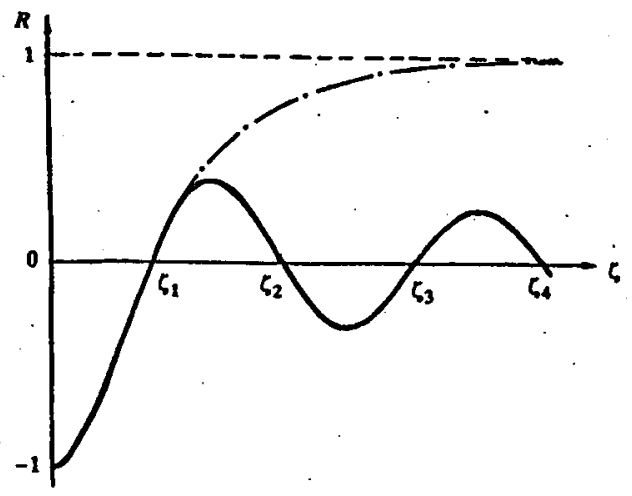

Fig. 2. The bound component of the electron wave function reflection coefficient $R$ vs. the field parameter $\zeta$.

An additional information about the stabilization-destabilization regimes can be deduced from an analysis of the reflection coefficient $R$ in the bound component of the strong-field electron quasi-energy function. In the theory presented here this reflection coefficient is shown in Fig. 2 and analytically is given by

$$
R(\zeta)=-\exp (-2 \mathrm{i} \mu)=-J_{0}(2 \zeta) .
$$

At the points $\zeta=\zeta_{\mathrm{i}}(i=1,2, \ldots) R(\zeta)=0$. This is the case of the most fast ionization when each portion of the electron wave function entering the quasiclassical region $r<r_{\mathrm{q}}$ makes an almost immediate and complete transition to the continuum. The regions where $R(\zeta)>0$ correspond to a partial stabilization which however is incomplete because $R_{\max }<1$, i.e., at any $\zeta \neq 0$ at least a part of the electron population is transferred to the continuum after a single crossing of the quasiclassical region. In the "old" strong-field theory $R(\zeta)$ would approach 1 when $\zeta \rightarrow \infty$ (the dash-dotted line at Fig. 2). This inversion of the reflection coefficient would explain the earlier investigated $[2,9]$ time-space structure of the bound part of the strong-field electron wave function. The main difference from this result arising in a "new" theory is an incomplete achievable inversion of the reflection coefficient and, hence, incomplete stabilization of the strong-field-driven atom.

At last, returning again to the ionization time $T_{\mathrm{i}}\left(\varepsilon_{0}\right)$ discussed above, we would like to formulate an additional hypothesis concerning its strong-field behavior. The expected result is $T_{\mathrm{i}}\left(\varepsilon_{0}\right) \sim t_{\mathrm{K}} \sim 1 / \Delta E$ where $\Delta E=E_{n+1}-E_{n}=$ $E^{(n+1)}-E^{(n)}$ is the spacing between the closest quasi-energy levels. In fact, 


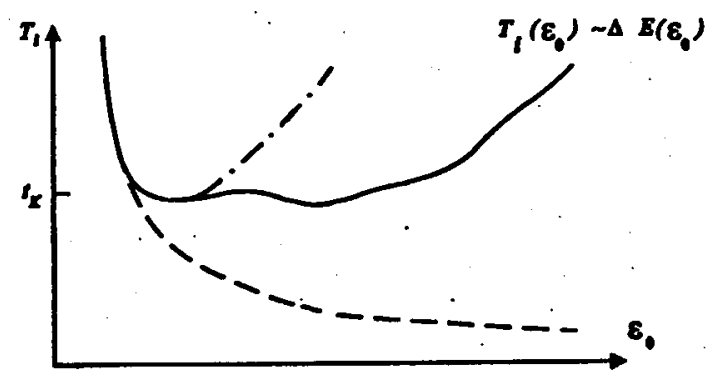

Fig. 3. The expected dependence of the ionization time $T_{\text {imin }}$ on the field strength $\varepsilon_{0}$.

the relation $\left[T_{\mathrm{i}}\left(\varepsilon_{0}\right)\right]_{\min } \sim 1 / \Delta E\left(\varepsilon_{0}\right)$ looks so natural that it can be even much more general than the presented theory itself. Then we can expect that in a very strong field $T_{\mathrm{i}}\left(\varepsilon_{0}\right)$ can grow because of a decreasing spacing between the closest strong-field quasi-energy levels (Fig. 3). Such a possibility correlates with the behavior of the strong-field atomic quasi-energy levels calculated in the so-called Kramers-Henneberger model [10]. Therefore, the hypothesis is that the decrease in $\Delta E\left(\varepsilon_{0}\right)$ is the main reason of growth of $\left[T_{i}\left(\varepsilon_{0}\right)\right]_{\min } \sim 1 / \Delta E\left(\varepsilon_{0}\right)$ and of the super-strong-field stabilization of atoms.

\section{Conclusion}

In a conclusion, let us describe briefly the main directions in which the presented theory has to be extended. First, as mentioned above, in the case of small frequencies $\omega$ one has to remove the used simplification under which all the transitions between the quasi-energy state $\chi_{\gamma}$ and lower lying states $\chi_{\gamma-n \omega}$ were ignored. In other words the sum over $n$ in Eq. (14) has to be extended to include negative $n$. The main question is how does this change affect the dispersion equation and the complex quasi-energies described above?

Second, a very large attention has to be paid to the ignored above angular motion of the electron. Even if the used above approximation of a "frozen" angular motion is good enough, the derived quasi-energies $\gamma$ depending parametrically on the angle $\theta$ (via $\varepsilon_{0}$ in fact equal to $\varepsilon_{0} \cos \theta$ ) have to be considered as an analog of the potential energy for a slow angular motion. The corresponding angular part of the Schrödinger equation has to be solved explicitly, and then the used above condition $\left\langle\widehat{l}^{2}\right\rangle \omega^{2 / 3} \ll 1$ has to be checked.

Third, a very important question concerns a relation between the eigenvalue and the initial value problems. To describe properly the time evolution of atomic populations one has to solve the initial value problem, probably under the same assumptions as used above in the solution of the eigenvalue problem. In such a theory the strong-field ionization time $T_{i}\left(\varepsilon_{0}\right)$ can be found explicitly, as it has been done in the "old" theory of interference stabilization $[2,9]$.

And the last, forth, strategically probably the most important problem mentioned above is a unification of different models of the field-induced stabilization, establishing of links between them. From this point of view, it seems to be rea- 
sonable and even necessary to try to check both numerically and analytically the formulated above hypothesis that the most general strong-field relation between the ionization time and spacing between the closest quasi-energy levels has a very simple form $\left[T_{i}\left(\varepsilon_{0}\right)\right]_{\min } \sim 1 / \Delta E\left(\varepsilon_{0}\right)$.

\section{References}

[1] See the papers published in the special issue of the journal Laser Physics on Atoms, Ions and Molecules in a Strong Laser Field, 3, (1993) \#2.

[2] M.V. Fedorov, Laser Phys. 3, 219 (1993).

[3] I.Ja. Bersons, Phys. Lett. A 84, 364 (1981).

[4] N.B. Delone, S.P. Goreslavski, V.P. Krainov, J. Phys. B 16, 2369 (1983) and 22, 2941 (1989).

[5] L.D. Landau, E.M. Lifshitz, Quantum Mechanics, Pergamon Press, Oxford 1977.

[6] I. Bersons, J. Opt. Soc. Am. B 7, 617 (1990).

[7] I.S. Gradstein, I.M. Ryzhik, Table of Integrals, Series and Products, Academic Press, New York 1980.

[8] M.Yu. Ivanov, in: Elementary Processes in a Laser Radiation Field, Spectroscopy Council of the Academy of Sciences, Moscow 1988, p. 33 (in Russian).

[9] M.V. Fedorov, M.Yu. Ivanov, A.M. Movsesian, J. Phys. B 23, 2245S (1990). 